\title{
The Application Accuracy of the Frameless Implantable Marker System and Analysis of Related Affecting Factors
}

\author{
Qinghang Li , Lucia Zamorano, Zhaowei Jiang, Fernando Vinas and Fernando Diaz
}

Wayne State University, Department of Neurological Surgery, 4160 John R. Suite 930, Detroit, MI 48201.

E-mail: li@neurosurg.wayne.edu. $\quad F a x:$ (313) 966-0368

\section{ABSTRACT}

The purpose of this study was to determine the application accuracy of a new frameless marker system for interactive intraoperative localization of intracranial lesions. The influence of image quality, registration error, repeatability, and marker distribution on the application accuracy were analyzed and compared. A phantom was mounted with the standard Z-D ring and also implanted with frameless marker system, which randomly distributed on the surface. The phantom was scanned as routine with $1 \mathrm{~mm}$ and $2 \mathrm{~mm}$ sections. The pixel sizes were used $1.18 \times 1.18$ and $0.59 \times 0.59$. The two systems were tested under different image quality and registration. The target point was digitized and the coordinates were recorded and compared with reference points. The difference between two systems were tested with paired t-test. Image data were loaded into a SUN Workstation and registered with NSPS.4.0 software. The coordinate of each fiducial marker was recorded into a file as the reference. The tip of each semi-invasive fiducial marker was digitized to achieve a frameless transformation matrix, and the special points on the Z-D ring were digitized to achieve a frame-based transformation matrix. The differences from the reference points were used as the deviation from "true point". The mean square root (RMS) was calculated to show the sum of vectors. The results of $2 \mathrm{~mm}$ section group showed that the registration error of frame-based system is $3.42 \pm 0.22 \mathrm{~mm}$ and the error of the frameless system is $1.01 \pm 0.63 \mathrm{~mm}(\mathrm{P}<0.001)$. The RMS are $2.57 \pm 0.54 \mathrm{~mm}$ and $\mathrm{I} .53 \pm 0.65 \mathrm{~mm}$ respectively $(\mathrm{P}<$ 0.001 ). The RMS of error registration (one point off 5 $\mathrm{mm}$ ) are $5.01 \pm 0.26 \mathrm{~mm}$ and $2.23 \pm 0.13 \mathrm{~mm}$ respective $(P=0.003)$. The results of $1 \mathrm{~mm}$ section gtoup showed that the RMS are $1.20 \pm 0.42 \mathrm{~mm}$ and $0.90 \pm 0.47 \mathrm{~mm}$ respectively $(\mathrm{P}=0,121)$. The higher the quality (the thinner scan thickness) of image it is, the better the application accuracy will be $(P=0.001$ and 0.032 respectively). These preliminary results showed that the frameless semi-invasive fiducial marker system can provide clinical acceptable accurate localization as the frame based surgical localization system did. There is no significant difference between the experimental and clinical results. The higher the quality of image it is, the better the application accuracy will be. But there is no significant difference between $1 \mathrm{~mm}$ sections and $3 \mathrm{~mm}$ sections of MRI images.

Key words: Image guided surgery, Application accuracy, stereotactic surgery, frameless and frame based systems,

\section{INTRODUCTION}

Stereotactic localization using multimodality of medical images (such as CT or MRI) provides highly accurate intraoperative localization and allows the surgeon to resect a lesion in its entirety while sparing critical areas of the brain. The use of stereotactic frames and rigid pin fixation to the skull have provided extreme levels of mechanical sccuracy for surgical localization. As an alternative to conventional stereotaxis, a variety of frameless stereotactic systems have been developed to provide surgical localization intraoperatively, which has eliminated the requirements of a rigid frame that may interfere with access to the operating field (1,3-5). As the use of computer-interactive technologies have become more common in neurosurgery, frameless methods are also widely applied to the neurosurgery and the need for an optimum skull fiducial marker system has increased. Two main fiducial marker system have been developed. Skin marker system is easy to use and sticks on the surface of the skull. However, the motion of the skin during the surgery or other procedures is an important source of affecting factors on the application accuracy. The bone screw marker system is fixed on the skull and more stable during the surgery. It can provide more reliable results for clinical usage. Therefore, it is very important to have a quantitative study to analyze the affecting factors on the application accuracy.

The application accuracy of a surgical localization system is a function of its mechanical accuracy interacting with the selected parameters of imaging studies chosen to visualize the lesion and its related anatomy. However the mechanical accuracy of the system can not represent the application accuracy of this system. It will be significantly worse than the weakest link of localization accuracy in a system, because other errors will be added to it rather than being hidden within it. In the surgical procedure artificial 
factors are also an important sources of the errors of localization. In addition to the mechanical limitations of the surgical localization system, the errors associated with the many steps of surgical localization (including imaging techniques, point selection, vector calculation, mechanical couplings and adjustments, other system adjustments, registration procedure and techniques, etc.) all contribute to the final clinically relevant error. In order to provide high levels of surgical localization accuracy, all of these error related factors should be controlled and reduced to a extreme low level. Therefore to determine how much distortion of these affecting factors caused during the whole procedure of image guided surgery on the application accuracy of the system is a very important content for this study. Theoretically speaking, the frame-based and frameless surgical localization system should have similar application accuracy. However quantitative comparison between these two system has not been reported. We present our preliminary experience with the use of semiinvasive fiducial markers (Fisher, Freiburg-Leibinger) comparing their application accuracy to that of the ZD stereotactic ring (Fisher-Leibinger, Freiburg, Germany). for the interactive-intraoperative localization of intracranial lesions.

\section{MATERIAL AND METHODS}

This study was designed to determine the influence of different affecting factors on the application accuracy. The each affecting factor were tested and analyzed. A paired $t$-test was used to perform statistical analysis

\subsection{Experimental Setup}

The semi-invasive markers developed with FisherLeibinger consist of three parts: a titanium screw, a base, and an insert. Due to the varying thickness of the galea, the titanium bone screws are available in two different lengths, 10 and $18 \mathrm{~mm}$, both with a thread length of 7 $\mathrm{mm}$. The base has in one end a thread to attach to the screw, and a cavity for insert at the opposite end. There are three types of inserts available: a computed tomographic (CT) -angiogram compatible insert, a multimodality image insert, and an intraoperative insert. The CT-angiogram insert is a gold-filled sphere. The multimodality image insert can be filled with different substances, including radioactive isotopes for use with CT, magnetic resonance imaging (MRI), digital angiography (DA), or positron emission tomography (PET) scans. The intraoperative insert has been created to replace the image insert for intraoperative registration. The image inserts are spherical in shape so the center is not accessible for intraoperative registration while the intraoperative insert is flat, and its height corresponds exactly to the center of the spherical image insert

\subsection{Phantom preparation}

A phantom was mounted with the standard Z-D ring and also implanted with five semi-invasive screws of the frameless marker system, which randomly distributed on the surface. A steel sphere with diameter of $2 \mathrm{~mm}$ was mounted into the phantom as target point. The Phantom was imaged using a Semens Somatom Plus CT scanner ( Siemens, Eriangen, Germany ). Scan thickness was set at 1 and $2 \mathrm{~mm}$; for each thickness, two kinds of image resolution were used. Pixel sizes are 1.18 $\times 1.18$ and $0.59 \times 0.59$ respectively. We use $2 \mathrm{~mm}$ thickness and pixel size $1.18 \times 1.18$ image resolution in one group, and $1 \mathrm{~mm}$ thickness and $0.59 \times 0.59$ image resolution in another group to observer if there is a significant difference between the best and the worst image quality conditions. The images were transferred to a stereotactic computer. The Neurosurgical Planning System (NSPS) software developed at Wayne State University was used for image registration. The NSPS runs on a SUN SPARC10 station (Sun Microsystems, Mountain View, California), used to achieve fast calculations to reconstruct volumes from imaging studies. The workstation is connected to the Detroit Medical Center's central computer network, from which images derived from $\mathrm{CT}$ and MRI can be transferred. It allows image viewing and manipulation in real-time during preplanning and intraoperatively. The coordinates of target point were recorded and saved into a file as a reference point.

\subsection{Surgical localization system}

A contact-less, pre-calibrated motion analysis system was chosen to locate the instruments. It can track up to 256 pulsed infrared light emitting diode (LED) markers with a maximum sampling rate of about 3500 markers per second (OPTOTRAK 3020, Northern Digital, Waterloo, CAN). Within a field of view of $1.0 \mathrm{~m}$ $x 1.2 \mathrm{~m}$ at a distance of $2.0 \mathrm{~m}$ the camera can locate each LED with an accuracy of $0.15 \mathrm{~mm}$. By attaching at least three markers onto each rigid body its location and orientation in space can be determined. The system is precalibrated, reducing set-up time and allowing the better tolerance to environmental changes. The limitation of the system is the need of a direct viewing between the camera and the LED's. To overcome this limitation we have designed a special holder to allow for different heights and angulations. The OPTOTRAK system has the ability to define several rigid bodies. Each rigid body is assigned a separate dynamic reference. It provides 
relative coordinates from the camera to any one of these rigid body's dynamic reference on the fly, by attaching to the skull or other rigid body. By calibrating the relative coordinates the transformation matrix is established. This eliminates the need for new matching during neurosurgery procedure. The motion analysis system is controlled by DOS based software running on a personal computer networked with a Unix workstation (SUN Microsystems, Inc. Mountain View, CA) which is used for image data acquisition/reconstruction and real-time instrument visualization. A single monitor provides all information.

\subsection{Experimental procedure}

In order to determine the influence of the quality of image, a special designed plate mounted with ten semiinvasive maskers was used. The distance between each marker was directly measured by a precise vernier calliper. The scan section thickness were $1 \mathrm{~mm}$ and 3 $\mathrm{mm}$ respectively. After images were transferred into SUN Workstation, NSPS.4.0 software was used to reconstruct the images to form 32-D images (i.e. axial, coronal, and sagittal planes). Then, each marker coordinate was digitized 8 times and recorded into a file. The distance between markers from digitized results were compared with the distance that from directly measured. A paired t-test was used to compare the results from two different image quality groups and also from different distance groups.

For each image group, the experiment procedure included the image registrations, which based on the Z-D ring and frameless marker system and the digitization of the target point. In each image group, three times of image registration were finished in order to check the repeatability of the system. In addition, an artificial error registration was also finished by $5 \mathrm{~mm}$ differ from the ideal point of the registration in order to observe the influence of artificial error during the image registration. In each registration, the target point was digitized 3 times. The coordinates of each digitization were recorded and save into a file for the off-line statistical analysis. These coordinates were used to compare with the coordinates of the reference point.

\subsection{Image Data Acquisition}

Interactive image guidance requires the registration of images derived from $\mathrm{CT}$ and/or MRI. Those images must be registered to each other and to the patient by using common points of reference, such as anatomical points or fiducial markers.
Before Gadolinium enhanced MRI, we placed five semi-invasive markers (Leibinger-Fisher, Freiburg, Germany) on the phantom. The semi-invasive markers consist on two parts: a base, which is fixed to the head with a very small $(2 \mathrm{~mm}$ ) Titanium screw, and the insert. The threads of the base were firmly screwed to the skull bone, using the pilot hole as a guide. The insert is a $3 \mathrm{~mm}$ ball-shaped plastic container, filied with a radio-opaque material, which was designed to be brightly visible on both CT and MRI images, including T-1, proton density, and T-2 weighted images. Once placed, these markers remained on the phantom during the scanning, and thorough the experimental procedure.

In order to determine the influence of the quality of image, a special designed plate mounted with ten semiinvasive markers was used. A MRI T1 sequence was used and the scan section thickness were $1 \mathrm{~mm}$ and 3 $\mathrm{mm}$ respectively.

\subsection{Intraoperative registration and localization}

Registration is the process of defining some special points based on the fiducial markers or anatomical landmarks from CT, MRI, or PET scan data and correlating them with these points located on the head of the patient in the real world, in the operating room. The goals are: firstly, to match, or correlate, data from the medical image to the 'real worid', which refers to the coordinate space of the surgical instruments. A tracking device is attached to the instruments to continually relay information regarding position to the system. Coordinate matching ensures that any point seen in a medical image corresponds to an actual point in the real world (i.e., the patient's spinal anatomy). Second is to correlate different images with each other. Each imaging modality displays anatomical structures and lesions in a unique way. This benefits the surgeon by providing several different ways to viewing a same anatomical structure, but requires the development of an interactive relationship between the images and the 'real world'. Registration is used to build the relationship and enable the surgeon to use each imaging modality to its greatest advantage for localizing the anatomical structure. Intraoperative digitization through an infrared system is based upon the use of a three-dimensional (3-D) motion analysis system (OPTOTRAK, Northern Digital, Waterloo, Canada). This system is consists of an opto-electronic system with light- emitting diodes (LED). Three infrared sensors track target points defined by several miniature leads mounted on the surgeon's operative instrument, in a predefined relationship, and referred to as a "surgical rigid body or pointer". Another similar rigid body is mounted to the head bolder or patient's ring (patient's 
rigid body). The patient's rigid body is used to tell the system where the real patient is, and the surgical rigid body tells the system the position of the surgical instrument. Then transformation matrix are calculated, the unique coordinate system is set up. Each time the surgeon moves the instrument, three monitors mounted in the room display the instrument's position on previously acquired computer-generated images.

In the image registration the image coordinates of each fiducial marker obtained from the CT scan was recorded into a file as the reference points (absolute image coordinates). In the operating room, the image registration was performed for the semi-invasive fiducial markers and the Z-D stereotactic ring. By touching the tip of each semi-invasive fiducial marker and four points on the Z-D ring to form the appropriate transformation matrix for each system. The image coordinates are matched with the real world patient's anatomy. Framebased registration was showed in workstation $A$ and frameless registration was showed in workstation $\mathbf{B}$. After the image registration the surgical tracking mode was used to track the surgical instruments. By touching the tip of each semi-invasive fiducial marker with the pointer (surgical rigid body), the system recorded their spatial position, as they were touched by the tip of the probe during the image registration. In this way, the $X$, $\mathrm{Y}$ and $\mathrm{Z}$ coordinates of the markers were recorded.

\section{7._Statistical_analysis}

A systematic error analysis was performed comparing the coordinates of target points on the medical images and the coordinates of intraoperatively digitizing target points based on the registration from each surgical localization system. Statistical analysis was performed on the sample of experimental data and sample of 19 patients.

A comparison of the 3-D measurements $(x, y, z)$ of the coordinates of the target point from each experimental data and each patient with the ring and fiducial markers to absolute image coordinates was performed. The ten times repeated measurements for each test using each of method, which allowed for testing of internal consistency of the measurements for each method. Statistical analysis pertaining to various comparisons among methods was performed on the average measurements per study subject.

The mean deviation from the absolute image coordinates were calculated from all $3-D$ mean measurements $(x, y, z)$ of each experiment data and patient for each methods correlating the ring and fiducial markers-based registration to the absolute image coordinates. Also, the deviation from target point in three direction (anterior-posterior, medial-lateral, and superior-inferior direction) of each experimental data and patient between ring and fiducial measurements were calculated similarly. Therefore, to assess differences among patients per each of the $x, y, z$ measurements a mean square root of the distance between the planes of the three mentioned distances, for the $i t h$ patient, was calculated as follows:

$$
\begin{gathered}
\text { distance }_{i j k}=\operatorname{SQRT~}\left(\left(X_{i j}-X_{i k}\right)^{2}+\left(Y_{i j}-Y_{i k}\right)^{2}+\left(Z_{i j}-Z_{i k}\right)^{2}\right) \\
\text { where } j, k=1,2,3 .
\end{gathered}
$$

A paired t-test was applied to test for significance of the mean deviation between the frame based and frameless system. A one-sample mean t-test was applied to test for mean significance of distance among the three methods. A paired t-test was used to compare the magnitude of difference of mean distances (Delta values) of the ring-based registration and absolute image coordinates versus the fiducial markers-based registration and absolute image coondinates. To estimate the average mean difference of the distance, a $95 \%$ confidence interval $(95 \% \mathrm{CI})$ was obtained. The semi-invasive fiducial system was compared with the ZD stereotactic ring (Fisher, Freiburg, Germany), which has demonstrated a high level of application accuracy in previous studies. After registered by both system, 119 random points on these 12 patients were collected and compared. A statistical analysis was performed, comparing the error difference between both methods of registration (ring fiducial and the semi-invasive fiducial markers), in determining the position of ten points on the patient's head. For that purpose, we compared the Mean Root Square (RMS), the Mean Error of Localization (MEL), and the Mean Error in 3-D (MED).

The RMS represents the mean of the sum of vectors between two records on one points which is the maximum distance between them. It is calculated as follow:

$\mathrm{RMS}=\mathrm{SQRT}\left((\mathrm{X} 1-\mathrm{X} 2)^{2}+(\mathrm{Y} 1-\mathrm{Y} 2)^{2}+(\mathrm{Z} 1-\mathrm{Z} 2)^{2}\right)$

The Mean error of localization (MEL) is determined by calculating the mean of the sum of the scalar distances between the true target and the points achieved by the localization system. This number is based on how far from target each individual attempt was, irrespective of direction. Here we assume that records from Z-D stereotactic frame system as the true target. The mean error of localization takes into account the precision of each individual localization attempt. In this way, the 
surgeon can reasonably know " what is the greatest distance that this particular device could place me away from my chosen target $"$ ? It is calculated as follow:

$M E L=((X 1-X 2)+(Y 1-Y 2)+(Z 1-Z 2)) / 3$

The Mean error in 3-D (MED): Using this way is to analyze the error rate in three different dimensions. It reflects the error on a specific dimension, therefore it should be calculated for each axis by the formula

$$
\mathrm{MED}=(\mathrm{X} 1-\mathrm{X} 2) / \mathrm{N}
$$

\section{Results}

\subsection{The error produced by image and digitization}

This affecting factor is defined as the difference between a known distance (precisely measured before the scan procedure) and same distance digitized from medical image. The etror is related with the scanner and human digitization. The results are shown in Table 1:

Table 1: The errors produced by image scanner and human digitization in different image quality groups (Mean error \pm SD mm)

\begin{tabular}{|l|l|l|}
\hline Distances $\backslash$ Sections & $1 \mathrm{~mm}$ & $3 \mathrm{~mm}$ \\
\hline $25 \mathrm{~mm}$ & $0.17 \pm 0.19$ & $0.20 \pm 0.21$ \\
\hline $50 \mathrm{~mm}$ & $0.19 \pm 0.16$ & $0.25 \pm 0.21$ \\
\hline $75 \mathrm{~mm}$ & $0.27 \pm 0.24$ & $0.21 \pm 0.14$ \\
\hline $100 \mathrm{~mm}$ & $0.21 \pm 0.19$ & $0.24 \pm 0.24$ \\
\hline
\end{tabular}

There is no significant differences between $1 \mathrm{~mm}$ and $3 \mathrm{~mm}$ sections of MRI groups. In varying the distances, there is also no significant difference. For a surgical localization system, this is not a important affecting factor to the application accuracy of the system.

\subsection{The application accuracy of the frame-based and frameless surgical localization system}

Anteroposterior, lateral, and vertical components of the target coordinate from each digitization were examined individually for their error. The Root-MeanSquare (RMS) was also calculated from these three components. The results are shown in Table 2 and Table 3. There is a significant difference between two systems $(P=0.0001)$ in low resolution and $2 \mathrm{~mm}$ thickness scan images but no significant difference in high resolution and $1 \mathrm{~mm}$ thickness image groups $(P=0.121)$. The image quality also takes a important role on the application accuracy. The higher the quality (the thinner of the scan thickness) of image it is, the better the application accuracy it will be $(P=0.001$ and 0.032 respectively) (Figure 1)

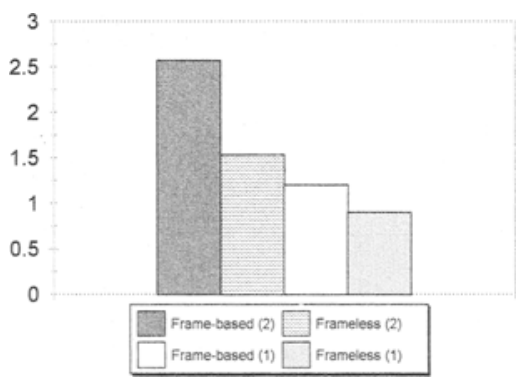

Figure 1. The comparison of application accuracy between frame-based and frameless localization system. $(2=2 \mathrm{~mm}$ thickness of CT scan and $1=1 \mathrm{~mm}$ thickness of CT scan

Table 2: Comparison of application accuracy between frame-based and frameless localization system ( Mean error \pm SD mm, CT sections $=2 \mathrm{~mm}$ )

\begin{tabular}{|l|l|l|}
\hline & $\begin{array}{l}\text { Frame-based } \\
\text { system }\end{array}$ & $\begin{array}{l}\text { Frameless } \\
\text { system }\end{array}$ \\
\hline Anteroposterior & $1.20 \pm 0.29$ & $0.76 \pm 0.31$ \\
\hline Lateral & $1.79 \pm 0.17$ & $1.03 \pm 0.48$ \\
\hline Vertical & $1.33 \pm 0.89$ & $0.45 \pm 0.38$ \\
\hline RMS & $2.57 \pm 0.54$ & $1.53 \pm 0.65$ \\
\hline
\end{tabular}

Table 3: Comparison of application accuracy between frame-based and frameless localization system ( Mean error $\pm \mathrm{SD} \mathrm{mm}, \mathrm{CT}$ sections $=1 \mathrm{~mm}$.)

\begin{tabular}{|l|l|l|}
\hline & $\begin{array}{l}\text { Frame-based } \\
\text { system }\end{array}$ & $\begin{array}{l}\text { Frameless } \\
\text { system }\end{array}$ \\
\hline Anteroposterior & $0.34 \pm 0.26$ & $0.67 \pm 0.41$ \\
\hline Lateral & $0.48 \pm 0.26$ & $0.45 \pm 0.32$ \\
\hline Vertical & $0.98 \pm 0.45$ & $0.3 \pm 0.12$ \\
\hline RMS & $1.20 \pm 0.42$ & $0.90 \pm 0.47$ \\
\hline
\end{tabular}


3.3. The influence of artificial errors in the image registration

During surgical procedure if an artificial error in the image registration happened, what kind of effects will be occurred on the application accuracy. In this experiment, a $5 \mathrm{~mm}$ deviation from ideal registration point was used as registration point. These results are shown in Table 4 and 5.

Table 4: Influence of artificial error on the application accuracy (Mean error \pm SD , CT sections $=2 \mathrm{~mm}$ )

\begin{tabular}{|l|l|l|}
\hline & $\begin{array}{l}\text { Frame-based } \\
\text { system }\end{array}$ & $\begin{array}{l}\text { Frameless } \\
\text { system }\end{array}$ \\
\hline Anteroposterior & $1.95 \pm 0.17$ & $0.93 \pm 0.42$ \\
\hline Lateral & $1.16 \pm 1.14$ & $1.58 \pm 0.81$ \\
\hline Vertical & $2.85 \pm 0.43$ & $0.64 \pm 0.36$ \\
\hline RMS & $3.91 \pm 0.66$ & $2.01 \pm 0.73$ \\
\hline
\end{tabular}

Table 5: Influence of artificial ert on application accuracy (Mean error \pm SD $\mathrm{mm}, \mathrm{CT}$ sections $=1 \mathrm{~mm}$.)

\begin{tabular}{|l|l|l|}
\hline & $\begin{array}{l}\text { Frame-based } \\
\text { system }\end{array}$ & $\begin{array}{l}\text { Frameless } \\
\text { system }\end{array}$ \\
\hline Anteroposterior & $1.11 \pm 0.51$ & $0.39 \pm 0.53$ \\
\hline Lateral & $0.77 \pm 0.81$ & $0.63 \pm 0.48$ \\
\hline Vertical & $2.42 \pm 0.93$ & $0.61 \pm 0.37$ \\
\hline RMS & $2.92 \pm 1.23$ & $1.15 \pm 0.07$ \\
\hline
\end{tabular}

The artificial errors during the image registration can also directly effect on the clinical application accuracy in the both systems. There is a significant difference between normal registration and artificial error registration in two image quality groups. $(P=0.001$ and 0.001 respectively ) The frameless localization system has the better application accuracy in the two image quality group. ( $P$ $=0.029$ and 0.04 respectively) (Figure 2 ).

\subsection{The influence of marker's position on the application accuracy}

Generally speaking, markers of frameless system should be distributed as far as possible for the

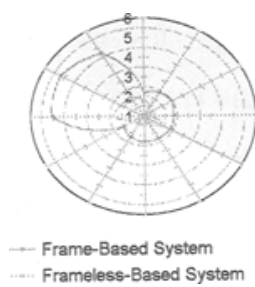

Figure 2. The influence of artificial error on the application accuracy.

reason of accuracy. However, it is difficult to determine what kind of distribution is the best. We tested two groups of distributions. One is localized around the area $6 \times 6 \mathrm{~cm}$, which is close to surgical area and easy to do the registration. Another group is distributed around the whole skull, which is suppose to be more accurate than the local distributed marker group. The results are shown in Table 6 and 7.

Table 6: Influence of marker distribution on the application accuracy

(Mean error $\pm S D \mathrm{~mm}, C T$ sections $=2 \mathrm{~mm}$ )

\begin{tabular}{|l|l|l|}
\hline & $\begin{array}{l}\text { Local } \\
\text { distribution }\end{array}$ & $\begin{array}{l}\text { Whole skull } \\
\text { distribution }\end{array}$ \\
\hline Anteroposterior & $1.01 \pm 0.44$ & $0.93 \pm 0.42$ \\
\hline Lateral & $0.47 \pm 0.54$ & $1.58 \pm 0.81$ \\
\hline Vertical & $0.41 \pm 0.26$ & $0.64 \pm 0.36$ \\
\hline RMS & $1.33 \pm 0.37$ & $2.01 \pm 0.73$ \\
\hline
\end{tabular}

Table 7: Influence of marker distribution on application accuracy (Mean error \pm SD $\mathrm{mm}, C T$ sections $=1 \mathrm{~mm}$ )

\begin{tabular}{|l|l|l|}
\hline & $\begin{array}{l}\text { Local } \\
\text { distribution }\end{array}$ & $\begin{array}{l}\text { Whole skull } \\
\text { distribution }\end{array}$ \\
\hline Anteroposterior & $0.30 \pm 0.15$ & $0.39 \pm 0.53$ \\
\hline Lateral & $0.39 \pm 0.16$ & $0.63 \pm 0.48$ \\
\hline vertical & $0.17 \pm 0.21$ & $0.61 \pm 0.37$ \\
\hline RMS & $0.58 \pm 0.13$ & $1.15 \pm 0.07$ \\
\hline
\end{tabular}


The local distribution of markers around the lesion has the better application accuracy. $(P=0.001)$

\section{Discussions}

The first affecting factor in the image guided surgery is image factor. After images transformed from scanned and restructured how much distortion occurred? From our results, it seems very small only $0.2 \mathrm{~mm}$ to 0.3 $\mathrm{mm}$. This deviation generally will not produce a big infuence on the application accuracy of the system. The different distances from $25 \mathrm{~mm}$ to $100 \mathrm{~mm}$ produced a similar error. There is no significant difference between them. In $1 \mathrm{~mm}$ and $3 \mathrm{~mm}$ sections of MRI groups, it also showed a similar results with no statistically difference. However when thickness increased to $10 \mathrm{~mm}$, the difference is significant. Our results only proved that under $3 \mathrm{~mm}$ thickness of section, the application accuracy no significant improved as the thickness reduced.

Theoretically speaking, the frame-based and frameless surgical localization system should have similar clinical application accuracy because the transformation matrix from both systems are calculated by at least three fixed points on the Z-D ring or on the fiducial markers. This is based on rigid body mechanics. The only difference is the points from the Z-D ring are on the ring which have to be fixed to the skull by pins, while the points from fiducial markers are directly fixed to the skull by the screws.

Registration methodology constitutes a basic process in image-guided surgery. The interface surrounding the patient's head (i.e. stereotactic system or fiducial markers) must accurately transfer the target coordinates from $\mathrm{CT}$ and MRI to the digitizing system. Conventional stereotactic surgery uses rigid fixation (ring) mounted on the head of the patient by several screws (frame-based stereotaxis). Stereotactic frames have been the standard of clinically accepted levels of accuracy; consequently, any new methodology for registration should be compared against this basic standard. Although they are accepted from the accuracy point of view, the use of stereotactic nings offers several disadvantages. They are uncomfortable for the patient, and logistically they should be placed the same day of the surgery. Furthermore, the stereotactic ring can be displaced from its original position with the possibility of mechanical error on focalization, as we have observed in one of our study patients. The longer the ring remains placed on the patient, the risk of this complication is higher; special attention is required to avoid frame dislocation. Additionaliy, the use of a standard stereotactic ring complicates airway management during surgery, especially during awake procedures. Moreover, standard stereotactic rings are obstructing, limiting the choices of surgical approaches. This is especially true for skull base lesions. or when a combined supra- or infratentorial approach is required.

As an altemative to conventional stereotaxis, a variety of frameless stereotactic systems have been developed to provide surgical localization intraoperatively. They provide a reference interface, allowing for an unobstructed surgical approach and airway management. As of last year several frameless navigational devices have been developed $(2,5,6,7-12)$. Generally, two methods are currently used for frameless stereotaxis; fiducial markers applied to the scalp or semipermanent fiducial markers rigidly fixed to the patient's skull. When fiducial markers attached to the skin are used, the scalp may move relative to the cranial bones, deviating the fiducial points from their intended positions. These skin-attached fiducial markers are useful for some procedures, but do not provide the same level of accuracy obtained with rigid fixation. However, most frameless digitizing systems have been developed using noninvasive fiducial markers attached to the skin.

In order to achieve a higher degree of accuracy with frameless systems, a system of semipermanent fiducial markers has been developed. These markers could be left in place for several days without risk of being displaced, and allow for staged procedures, which are required in epilepsy surgery, for example, or in some skull base tumors.

In this study, we compared semipermanent fiducial marker accuracy with stereotactic frame accuracy, using the frame as the standard of a clinicallyacceptable degree of accuracy. Using the same image protocol, the statistical analysis revealed a small significant difference between both methodologies $(2.95$ $\pm .45 \mathrm{~mm}$ ). In order to define which of the two methods is more accurate, we compared each with the absolute image coordinates. Our study showed that the semipermanent fiducial marker system is more accurate than the stereotactic ring (the difference with the absolute image coordinates was $3.35 \pm .59 \mathrm{~mm}$ for the stereotactic ring, and $1.72 \pm .42 \mathrm{~mm}$ for the fiducial system). Although the difference between the two methodologies is statistically significant, its clinical implications may not be relevant.

An assessment of differences among the mean differences of various methods per each of the $x, y, z$ coordinates is done by constructing the $95 \% \mathrm{Cl}$. A mean 
difference of $Z_{2}-Z_{1}$ (Table 2) was significant, which implies that the significant difference in the distance is possibly due to this. Furthermore, the $95 \% \mathrm{CI}$ also provides an estimate of the size of $\square Z$ (i.e. $\mathrm{DZ}=\mathrm{Z}_{2}-\mathrm{Z}_{1}$ ) with a probability of $95 \%$. Therefore, fiducial-based registration is more accurate than the stereotactic frame.

We believe that the semi-permanent implantable fiducial marker system represents the next step in the application of stereotactic techniques in neurosurgery. It cannot yet replace traditional frame-based stereotactic surgery when minimal invasion and maximum prediction of target trajectory repeatability is required, like during a biopsy of a deep-seated lesion, functional procedures, or placement of implants. In other words, a function of stereotactic frames that cannot be replaced by the semipermanent fiducial system is its ability to act as an accurate holder and guidance to bring an instrument to a specific target. All frameless passive systems imply an iterative trial error method not acceptable for those procedures. However, this semi-permanent implantable fiducial system is ideal for a far greater range of cases in which frame-based stereotaxis is too limited, such as large centered craniotomies, skull base procedures, lesions where a combined supra-infratentorial approach, or staged procedures are required. In the future, active robotic systems could be used to guide an instrument to a target in a defined trajectory, which may be more precise than that achieved with the stereotactic arc. Interactive image-guided neurosurgery is evolving beyond the limitations placed by the mechanically-based stereotactic frames systems designed at the turn of the century. As new surgical navigational frameless systems are being developed, we anticipate that the semipermanent fiducial markers system are likely to replace other fiducial systems or even the stereotactic ring, introducing a new generation of possibilities in neurosurgery.

\section{References}

1. Bucholz RD, Smith KR (1993) A comparison of sonic digitizers versus light emitting diode-based location. In Maciunas RJ (ed) Interactive Image-Guided Neurosurgery. AANS Publications Committee, Park Ridge 1993. Pp 179-200

2. Bucholz RD, Ho HW, and Rubin JP.(1993) Variables affecting the accuracy of stereotactic localization using computerized tomography. J Neurosurg 79: 667-673

3. Golfinos JC, Fitzpatrick BC, Smith LR, Spetzler R (1995) Clinical use of a frameless stereatactic arm: Results of 325 cases. J Neurosurg 83: 197-205

4. Heilbrun MP, Mc Donald P, Wiker C, et al (1992) Stereotactic localization and guidance using a machine vision technique. Stereotact Func Neurosurg 58: $94-98$
5. Murphy MA, Barnett GH, Kormos DW, Waisemberger J (1993) Astrocytoma resection using an interactive frameless stereotactic wand: An early experience. J Clin Neurosci 1: 3337

6. Kato A, Yoshimine T, Hayakawa T, et al (1991) A frameless, armless navigational system for computerassisted neurosurgery. J Neurosurg 74: 845-849

7. Li Q.H., Holdener H.J., Zamorano L., King P., Jiang Z.W., and Diaz F.: Computer assisted insention of pedicle screws. Lecture Notes in Computer Science 1131. Eds by Karl Heinz $H_{-}$hne and Ron Kikinis, page 571-581, 1996.

8. Maciunas RJ, Fitzpatrick JM, Galloway RL, Allen GS Beyond stereotaxy: Extreme levels of application accuracy are provided by implantable fiducial markers for interactive image-guided neurosurgery. In Maciunas RJ (ed) Interactive Imaged-Guided Surgery. AANS Publications Committee, Park Ridge 1993, pp 259-270 9. Nolte LP, Zamorano L, Jiang Z, Wang Q, Langlotz F, Berlemann $U$ (1995) Image-guided insertion of transpedicular screws: A laboratory set-up. Spine 20: 497-500

10. Takizawa $T$ (1993) Neurosurgical navigation using a noninvasive stereoadapter. Surg Neurol 40: 1-7

11. Watanabe E, Mayanagi Y, Kosugi Y, Manaka S, Takakura K (1991) Open surgery assisted by the neuronavigator, a stereotactic, articulated, sensitive arm. Neurosurg 28: 792-800

12. Zamorano L, Nolte L, Jiang C, Kadi M (1993) Image-guided neurosurgery: Frame based versus frameless approaches. Neurosurgical Operative

Atlas 3: 402-422

13. Zamorano L, Nolte L, Kadi M, Jiang Z (1993) Interactive intraoperative localization using an infraredbased system. Neuro Res 15: 290-298

14. Zamorano L, Nolte LP, Kadi AM, Jiang Z (1994) Interactive intraoperative localization using an infraredbased system. Stereotact Funct Neurosurg 63: 84-88

15. Zamorano L, Kadi M, Jiang Z, Diaz FG (1994) Zamorano-Dujovny multipurpose neurosurgical imageguided localizing unit: Experience in 866 consecutive cases of "open stereotaxis". Stereotact Funct Neurosurg 63: 45-51 